resort community with an international, transient and disproportionally large young adult population. A local sexual health clinic operates at capacity. There is no provincial or national outreach CT screening campaign.

Methods Series of 15 outreach CT screening sessions, each 2-3 h duration, held in Whistler, BC, Canada in 2009 \& 2010. Sessions were held at resort staff-housing dinners, staff-housing lounge, entertainment, educational and sport events. Men and women $<30$ years were offered free CT nucleic acid amplification tests on urine. Positive cases were notified, with treatment and partner notification per standard of care. Primary outcome measures were age, gender and infection rates of outreach participants compared to $<30$ age cohort tested for CT at the sexual health clinic during same calendar years. Anonymous, post-test survey queried interval since last CT test, intention to test, health insurance, and satisfaction with the outreach experience. Unpaired t test $\& \chi^{2}$ analysis.

Results 112 tests for CT were obtained through outreach; 87.5\% response rate to post-test survey. Mean outreach age of 23.3 years was 14.4 months younger than comparison age cohort tested at clinic $(\mathrm{p}=0.0001)$. Males were tested at outreach in greater proportion than at clinic $(57.1 \%$ vs $46.5 \%, p=0.04)$. Proportion of asymptomatic cases was greater at outreach than clinic $(90 \%$ vs $46.6 \%, \mathrm{p}=0.01)$, yet positive test rates at outreach $(8.9 \%, 10 / 112)$ and clinic $(8.5 \%, 58 / 686)$ were comparable $(\mathrm{p}=0.87)$. On survey, $43.9 \%$ had never previously tested for CT, $53.7 \%$ were not already considering a test, $61.7 \%$ would not have gone for a test within the next 2 months. Only $27.6 \%$ had Canadian health insurance. $93.9 \%$ were satisfied or very satisfied with CT screening in an outreach setting.

Conclusions Intermittent, free, event-based outreach CT screening was operationally feasible, effective at increasing case detection, and highly acceptable to participants. Outreach attracted a younger age and more men than clinic. A large proportion of participants were first-time testers, over half were without prior intent to test or likelihood to test in near future, and most would have had to pay up-front for CT testing in a clinic setting. This study demonstrates both need and benefit of expanded CT screening efforts in the international resort setting.

\section{P5-S7.06 CHLAMYDIA SCREENING IMPLEMENTATION IN THE NETHERLANDS IS NOT COST-EFFECTIVE}

doi:10.1136/sextrans-2011-050108.601

${ }^{1} \mathrm{~B}$ Schmid, ${ }^{1} \mathrm{E}$ Over, ${ }^{1} \mathrm{I}$ van den Broek, ${ }^{1} \mathrm{E} O \mathrm{de}$ Coul, ${ }^{2} \mathrm{~J}$ van Bergen, ${ }^{3} \mathrm{CSI}$ Group, ${ }^{1} \mathrm{M}$ Kretzschmar, ${ }^{1} \mathrm{~A}$ de Wit. ${ }^{1}$ RIVM, Bilthoven, Netherlands; ${ }^{2}$ Soa Aids Nederland, Netherlands; ${ }^{3} \mathrm{CS} /$ Group, Netherlands

Background Chlamydia trachomatis is the most common sexually transmitted infection in western countries. Most infections are asymptomatic and may cause severe complications. In the Netherlands, 3 years of Chlamydia screening implementation (CSI) have been performed. Here, we model its cost-effectiveness after 10 years. Methods A cost-effectiveness analysis compares the relative costs and effects of two or more scenarios, and is usually expressed as the incremental cost-effectiveness ratio (ICER). The costs of the CSI program included those for hospital care, antibiotics, testing, and productivity loss. We measured the effects as either Major Outcomes Averted (MOA) or Quality Adjusted Life Years (OALY) gained. In the Chlamydia literature, MOAs usually consist of symptomatic pelvic inflammatory disease, chronic pelvic pain (CPP), ectopic pregnancy, infertility, and neonatal pneumonia. We calculated the ICER, the ratio of the above-mentioned costs and effects, for four scenarios: the default screening scenario (annual invitation of all 16-29 year olds), screening for women 16-29 only, for all 16-24 year olds, and biennial screening of all aged 16-29. To account for uncertainty in model parameters, we conducted a probabilistic sensitivity analysis (PSA).

Results If we compare the results of the four different scenarios presented in the abstract P5-S7.06 table 1, the default scenario has the most favourable ICER. This is probably due to the fact that the total number of invitations (and thus people tested) per year is the largest in the default scenario, implying that the fixed annual program costs are spread over more tests (and outcomes), which improves the ICER. The cost per QALY of all four scenarios seems acceptable if we include CPP in our OALY estimate. However, the evidence base for CPP forming nearly $90 \%$ of all OALYs lost is extremely weak. Therefore, we prefer the cost-per-OALY estimate excluding CPP, which is $30000-70000$. Considering previous decisions on population screening programs, this ratio is relatively high and cannot be regarded as cost-effective. However, these results should be interpreted with caution due to the weak evidence base for the disease progression model. Because of this, the PSA showed variability of the four ICERs of up to $50 \%$.

Abstract P5-S7.06 Table 1 Results from the economic CSI model

\begin{tabular}{|c|c|c|c|c|c|c|c|}
\hline Scenario: & $\begin{array}{l}\text { Total } \\
\text { costs } \\
\text { (M EUR) }\end{array}$ & $\begin{array}{l}\text { MOAs } \\
\text { averted }\end{array}$ & $\begin{array}{l}\text { OALYs } \\
\text { gained }\end{array}$ & $\begin{array}{l}\text { OALYs } \\
\text { gained } \\
\text { [w/o CPP] }\end{array}$ & $\begin{array}{l}\text { Costs/ } \\
\text { MOA } \\
\text { (k EUR) }\end{array}$ & $\begin{array}{l}\text { Costs/ } \\
\text { OALY } \\
\text { (k EUR) }\end{array}$ & $\begin{array}{l}\text { Costs/ } \\
\text { OALY } \\
\text { [w/o CPP] } \\
\text { (k EUR) }\end{array}$ \\
\hline Default & 6.9 & 1200 & 1600 & 210 & 5.6 & 4.3 & 32 \\
\hline Women only & 6.2 & 670 & 900 & 130 & 9.2 & 6.9 & 49 \\
\hline $16-24$ & 6.6 & 660 & 860 & 92 & 10 & 7.6 & 72 \\
\hline Biennial & 6.4 & 820 & 1100 & 140 & 7.9 & 6.0 & 47 \\
\hline
\end{tabular}

Conclusions We conclude that the evidence base for cost-effectiveness of Chlamydia screening is less strong than appeared from previous Dutch and foreign research, because of the much higher costs per MOA.

\section{P5-S7.07 CHLAMYDIA SCREENING IN CORNWALL: HOW OFTEN DO YOUNG PEOPLE GET RETESTED?}

doi:10.1136/sextrans-2011-050108.602

${ }^{1} \mathrm{~K}$ Turner, ${ }^{1} \mathrm{~L}$ Trela-Larsen, ${ }^{2} \mathrm{P}$ Gates, ${ }^{1} \mathrm{M}$ May, ${ }^{1} \mathrm{P}$ Horner. ${ }^{1}$ University of Bristol, Bristol, UK; ${ }^{2}$ Royal Cornwall Hospital Trust, Truro UK

Aim To analyse the uptake of screening and rescreening in Cornwall, UK within the National Chlamydia Screening Programme (NCSP). Methods We tested for any association between gender, age, test result and the probability of retesting. The time between tests was estimated using a Cox proportional hazards model and we tested whether the result of first test or gender influenced whether or not individuals were retested.

Results Between 2003-2009, 66513 tests in 46950 individuals were analysed. Most people were tested once. During this period the number of tests increased dramatically and the positivity declined (shown in Abstract P5-S7.07 table 1). Compared with those negative at the first test, positive cases were more likely to be retested and were retested sooner. Abstract P5-S7.07 table 1 Positivity among those tested in Cornwall 2003-2009.

Abstract P5-S7.07 Table 1

\begin{tabular}{lccccccc}
\hline & $\mathbf{2 0 0 3}-$ & $\mathbf{2 0 0 4 -}$ & $\mathbf{2 0 0 5 -}$ & $\mathbf{2 0 0 6 -}$ & $\mathbf{2 0 0 7 -}$ & $\mathbf{2 0 0 8 -}$ & \\
& $\mathbf{2 0 0 4}$ & $\mathbf{2 0 0 5}$ & $\mathbf{2 0 0 6}$ & $\mathbf{2 0 0 7}$ & $\mathbf{2 0 0 8}$ & $\mathbf{2 0 0 9}$ & \multicolumn{1}{c}{ Total } \\
\hline Tests & 4794 & 6153 & 11158 & 12864 & 14443 & 17101 & 66513 \\
Positive & 589 & 805 & 1086 & 1103 & 1213 & 993 & 5789 \\
Positivity & 0.1287 & 0.1376 & 0.1019 & 0.0901 & 0.0874 & 0.0604 & 0.0910 \\
\hline
\end{tabular}


Discussion We found that even in an area which has achieved high screening coverage, few people were re-screened. However, positive cases are more likely to be retested than negatives. This detailed county level analysis will provide information to policy makers to develop a long-term, sustainable and effective screening strategy.

\section{P5-S7.08 ASSESSMENT OF CHLAMYDIA TESTING COVERAGE USING HEDIS DATA: USA, 2009}

doi:10.1136/sextrans-2011-050108.603

G Tao, K Hoover, R Romaguera, C Kent. CDC, Atlanta, USA

Background In the USA, chlamydia screening of all sexually active women aged $\leq 25$ years is recommended, but screening rates are low. The Healthcare Effectiveness Data and Information Set (HEDIS) performance measure has tracked the time trend in screening since 2000. It has been found that among sexually active women aged $15-25$ years, chlamydia testing rates increased from $25.3 \%$ to $41.6 \%$ during 2000-2007. While rates have increased, they remain suboptimal, and it is unknown whether testing rates differ significantly between 2007 and 2009 or by the type of health plan.

Methods The 2009 HEDIS chlamydia testing dataset was analysed to estimate the annual coverage among sexually active women aged $15-25$ years who were enrolled in commercial health plans. Sexually active women were defined as those who had a claim or visit for pregnancy, contraception, cervical cancer screening, or STD diagnosis, screening, or treatment. A woman was counted as having a test if she had a claim or healthcare visit for any chlamydia test. Health plan types included in the HEDIS data are health maintenance organisations (HMOs), preferred provider organisation (PPO), point of service (POS), and any combination of $\mathrm{HMO}, \mathrm{PPO}$, and POS.

Results Of 2.4 million sexually active women, $54.5 \%$ were enrolled in $\mathrm{PPO}, 28.7 \%$ in combined $\mathrm{HMO} / \mathrm{POSs}, 13.8 \%$ in $\mathrm{HMOs}, 2.7 \%$ in combined $\mathrm{HMO} / \mathrm{POS} / \mathrm{PPO}$, and $0.3 \%$ in POSs. The overall annual chlamydia testing rate was $44.2 \%$ and significantly differed by plan type: $56.1 \%$ in $\mathrm{HMOs}, 45.7 \%$ in combined $\mathrm{HMO} / \mathrm{POSs}, 44.6 \%$ in POSs, 41.2 in combined $\mathrm{HMO} / \mathrm{POS} / \mathrm{PPO}$, and $40.5 \%$ in PPOs.

Conclusions The annual chlamydia testing rate continued to increase between 2007 and 2009 (41.6\% to 44.2\%). However, the testing rate remains suboptimal, suggesting that additional interventions are needed to further increase testing rates. The highest testing rates were found in $\mathrm{HMOs}$, healthcare settings that have interventions in place to promote provision of recommended healthcare services. The 2010 Affordable Care Act requires insurance plans cover preventive services such as chlamydia screening without patient cost sharing, and has the potential to increase chlamydia screening coverage throughout the US healthcare system. In light of these changes, it is important to continue to monitor chlamydia testing practices and to overcome barriers to testing.

\section{P5-S7.09 IS ABANDONING URETHRAL SMEAR MICROSCOPY FOR THE DETECTION OF NON-GONOCOCCAL NON-CHLAMYDIAL URETHRITIS IN ASYMPTOMATIC MEN A COST EFFECTIVE STRATEGY?}

doi:10.1136/sextrans-2011-050108.604

${ }^{1} \mathrm{O}$ Caffrey, ${ }^{2} \mathrm{~J}$ Saunders, ${ }^{2} \mathrm{C}$ Estcourt, ${ }^{3} \mathrm{R}$ Birger, ${ }^{3} \mathrm{P}$ White, ${ }^{1} \mathrm{~T}$ Roberts. ${ }^{1}$ University of Birmingham, Birmingham, UK; ${ }^{2}$ Oueen Mary University of London, London, UK; ${ }^{3}$ Imperial College London London, UK

Background Asymptomatic non-gonococcal non-chlamydial urethritis (NCNGU) is common and can only be diagnosed by urethral smear microscopy. UK guidelines no longer recommend urethral smear microscopy in asymptomatic men, leaving men with this condition and their sexual partners untreated. The clinical and economic significance of this is unclear. We do not know if the costs of microscopy screening for asymptomatic NCNGU outweigh any future health benefits. We performed a model based economic evaluation to compare a screening strategy which includes microscopy to one which omits microscopy in asymptomatic men. Methods The economic model was from the perspective of the UK health service and so only direct medical costs and outcomes are included. A hypothetical cohort of asymptomatic men who present at sexual health clinics, or at primary care is assumed to have Chlamydia trachomatis, Neisseria gonorrhoeae or NCNGU. In the economic model, untreated infections were taken into account for patients and their partners (pelvic inflammatory disease and tubal factor infertility in female partners). Probabilities and frequencies of these health events were informed by the outputs of a mathematical transmission model which used data from literature and national databases. A bottom-up costing estimated the cost for microscopy screening, while other cost inputs were sourced from published literature and online reference manuals for the economic evaluation. Appropriate sensitivity analyses were conducted to test the baseline results.

Results Currently, there are no robust quality-adjusted-life-year (OALY) data to value STI outcomes. Consequently, results will be presented as cost per major outcome avoided and cost per infection avoided, where major outcome averted refers to PID, infertility and tubal factor infertility. Incremental cost-effective ratios will be interpreted based on accepted precedents.

Conclusions The UK's current financial restraint has increased the importance of allocating resources based on need and value-formoney. This in turn, requires the UK health service to eliminate ineffective and inefficient services to create additional source of funding. Results from this study will indicate whether microscopy testing for asymptomatic men is good use of public money and whether funding for the service should be maintained.

\section{P5-S7.10 CHLAMYDIA SCREENING COVERAGE AMONG AMERICAN INDIAN AND ALASKA NATIVE WOMEN IN THE USA}

doi:10.1136/sextrans-2011-050108.605

S Tulloch, M Taylor. Centers for Disease Control \& Prevention, Albuquerque, USA

Background Racial and ethnic minorities are often disproportionately affected by health disparities. In 2009, American Indian and Alaska Natives (AI/AN) had the 2nd highest rates of chlamydia in the USA. In an effort to eliminate health inequities and reduce the burden of disease, the US Centers for Disease Control and Prevention (CDC) recommends annual chlamydia screening for all sexually active women $<25$ while reducing screening among older less at-risk individuals. We sought to assess and describe geographic regional differences in how recommendations have been adopted across Indian Country to identify what opportunities exist for improving care within the Indian Health Service (IHS) National STD Program's Stop Chlamydia Project, a screening program that partners with IHS/Tribal/Urban Indian health centers (I/T/U) to enhance and expand chlamydia screening among AI/AN

Methods We calculated chlamydia screening coverage rates and associated per cent positivity for women $<25$ and $>26$ years of age screened through sites participating in the Stop Chlamydia Project by geographic region. Screening coverage was calculated using the 2009 Indian Health Services facility-level user population estimates as denominator values.

Results Screening coverage among AI/AN women tested in Stop CT Project sites varied across geographic regions. Among women $<25$, rates ranged from $50.8 \%$ (Alaska) to $6.8 \%$ (California). Chlamydia 